\title{
Antibiotic Resistance, Uncertainty, and Medical Decision-Making by a Physician
}

\author{
Sanjana S. Batabyal \\ Department of Development Sociology, Cornell University, Ithaca, NY, USA \\ Email: ssb227@cornell.edu
}

How to cite this paper: Batabyal, S.S. (2018) Antibiotic Resistance, Uncertainty, and Medical Decision-Making by a Physician. Theoretical Economics Letters, 8, 2388-2399. https://doi.org/10.4236/tel.2018.811154

Received: June 30, 2018

Accepted: August 13, 2018

Published: August 16, 2018

Copyright $\odot 2018$ by author and Scientific Research Publishing Inc. This work is licensed under the Creative Commons Attribution International License (CC BY 4.0).

http://creativecommons.org/licenses/by/4.0/

\section{c) (i) Open Access}

\begin{abstract}
I explore the decision-making process of a physician in the context of otitis media-commonly known as an ear infection-when resistance to antibiotics is an issue. Otitis media provides a unique context in which to study such decision-making because there is no culture that one can use to definitively prove the presence of a bacterial infection. This creates an atmosphere in which the abuse of antibiotics is possible. I first use a decision tree to characterize the decision-making process and then I conduct numerical analysis using fictitious data to illustrate the working of my model. Next, I show how the dependence of the infection probability, $P_{\mathcal{S}}$ on socioeconomic variables can be used to shed light on a physician's behavior and on the patient-physician relationship. Finally, I discuss how the research presented in this paper might be extended in the future.
\end{abstract}

\section{Keywords}

Antibiotics, Behavior, Physician, Resistance, Uncertainty

\section{Introduction}

\subsection{The Problem}

Modern medicine has come a long way since the discovery of penicillin in 1928 by Alexander Fleming. Since the advent of this "miracle cure," there are now a wide variety of antibiotics at our disposal which physicians use regularly to cure individuals from all manner of bacterial infections. However, this ability to cure has not come without costs. Studies have found that Streptococcus pneumoniae, Streptococcus pyogenes, and Staphylococci, organisms that cause respiratory and cutaneous infections, and members of the Enterobactenaceae and Pseudornonas families, which cause diarrhea, urinary infection, and sepsis are now resistant to virtually all of the older antibiotics [1]. The matter of antibiotic resis- 
tance has become so extreme that scientists have even found strains of Streptococcus aureus, more commonly known as the strain of bacteria that causes "staph" infections, that are resistant to Vancomycin, which is considered to be one of the most potent antibiotics on the market [2].

Antibiotic resistance is not just costly to health; it also poses a financial cost. Both these costs have private and social aspects to them. Elbasha [3] recently estimated the annual loss associated with outpatient prescriptions for amoxicillin in the United States to be approximately US \$225 million. The danger that antibiotic resistance poses is so daunting that according to Fox [4], the World Health Organization's (WHO) director-general, Dr. Margaret Chan, has noted that antimicrobial resistance poses a fundamental threat to human health, development, and security.

A good example of a common condition where antibiotic abuse is a real risk is the case of an ear infection. Otitis media, commonly known as an ear infection, is a familiar ailment among young children. It is often caused by an upper respiratory viral infection such as the common cold or the flu but it can also be caused by bacterial infections such as sinusitis or pneumonia. Unlike other common ailments such as Streptococcal pharyngitis, more commonly known as "strep throat," there is no culture that can definitively confirm or deny the need to treat an ear infection with an antibiotic [5]. Thus, it is possible that physicians will prescribe antibiotics for an ear infection without knowing for certain whether that is the appropriate course of treatment. In the process, they unwittingly give rise to an environment that is conducive to the development of antibiotic resistance.

There are two commonly prescribed treatments for an ear infection. The first is "watchful waiting" where the patient is not prescribed an antibiotic and an individual such as a parent monitors the patient's condition. The second is the prescription of an antibiotic. The reader should understand that an ear infection is a very common ailment. In addition, this ailment provides a good setting in which to study issues pertaining to antibiotic resistance. Therefore, in this paper, I shall use this specific ailment to study medical decision-making by a physician. However, before I proceed to this paper's specific objectives, I shall first briefly survey the literature about antibiotic resistance.

\subsection{Literature Review}

The work of Amabile-Ceuvas et al. [6] and McKenna [7] shows that bacteria are becoming resistant to existing antibiotics at a much faster rate than we can find ways to control this resistance and that there is now a pressing need to find alternate therapies. The direct cause of antibiotic resistance, i.e., the overuse of antibiotics, is well documented. Indeed, antibiotic resistance now has fatal consequences. The Centers for Disease Control (CDC) estimate that at least two million people are infected and that 23,000 people die each year as a result of bacterial infections that are resistant to antibiotics [8]. 
Research regarding the behavior of physicians is generally more varied and less definitive than studies of antibiotic resistance. Studies have shown that $s o-$ cioeconomic variables are important in influencing a physician's drug prescribing habits. Kumar et al. [9] find that general practitioners serving populations experiencing "adverse social factors" such as low income, poor nutrition, and substance abuse are sometimes more likely to prescribe antibiotics because of the belief that such populations are more prone to bacterial infections.

Several studies have commented on the salience of the patient-physician relationship. Mangione-Smith et al. [10] point out that patient satisfaction is affected greatly by whether the patient feels that a physician has spent an adequate amount of time with him or her and by whether the patient has had a meaningful discussion about alternate treatment options with this physician. Butler et al. [11] examine the nature of prescription writing by general practitioners in England and point out that such doctors will often prescribe antibiotics unnecessarily in an attempt to maintain a positive relationship with patients. Fox [12] also demonstrates that doctors frequently prescribe antibiotics to keep patients happy.

The extant literature has paid insufficient attention to the nature of decision-making by physicians who prescribe antibiotics as part of a treatment regimen. Many of the existing studies point out that physicians are susceptible to social pressures and to patient expectations. For instance, Mangione-Smith et al. [10] note that the perceived pressure from a parent for a certain diagnosis for his or her child affects a physician's decision about whether to prescribe antibiotics. Bornstein and Emler [13] contend that psychological phenomena such as confirmation bias, the gambler's fallacy, hindsight bias, and anticipated regret all influence a physician's decision-making process. Other studies by Butler et al. [11], Kumar et al. [9], Macfarlane et al. [14], and Rollnick et al. [15] have collectively reinforced the salient point that patient expectations play a significant role in the decision-making process of a physician.

The studies discussed above have certainly increased aspects of our understanding of the drug prescribing behavior of physicians. Even so, with the exception of one paper $^{1}$ and despite the call for increased mathematical modeling by Manzo [16], to the best of my knowledge, there are virtually no studies in the literature that have mathematically analyzed medical decision-making by physicians in the presence of antibiotic resistance. Given this lacuna in the literature, my objective in this paper is to follow Manzo's [16] call and use the notion of a decision tree to shed light on medical decision-making by a physician who is faced with the issue of antibiotic resistance ${ }^{2}$.

${ }^{1}$ Batabyal and Batabyal [17] have recently provided a theoretical perspective on medical decision-making by patients and physicians. However, the modeling of the behavior of a physician in this paper is based on the theory of renewal-reward processes-see Ross [[18], 416-425]-and hence is abstract. In contrast, I show in a more straightforward manner how a decision tree can be used to shed useful light on medical decision-making by physicians.

${ }^{2}$ The standardization of the use of antibiotics is an important public policy issue but a discussion of this issue is beyond the scope of this paper. 
The remainder of this paper is organized as follows. Section 2.1 uses a decision tree to describe the nature of a physician's decision-making when antibiotic resistance is an issue to contend with. Section 2.2 conducts numerical analysis using fictitious data. Section 3.1 discusses the links between the dependence of a key infection probability $P_{S}$-further described below-on socioeconomic variables and on a physician's behavior. Section 3.2 sheds detailed light on the nature of the patient-physician relationship. Section 4 concludes and then discusses two ways by which the research in this paper might be extended.

\section{The Decision Tree}

\subsection{The Physician's Perspective}

Consider a mother who brings her daughter (the patient) to see a doctor (the physician). The mother suspects that her daughter has an ear infection. In my model, I suppose that the only way to determine whether there is, in fact, a bacterial infection is to treat the patient with an antibiotic ${ }^{3}$. Now recall that ear infections do not have either a culture or a test that can tell us whether the patient's condition is caused by either a bacterial or a viral infection. The physician must perform a medical examination prior to making a decision. The actions that follow from the medical examination are displayed in a decision tree (Figure 1). The outcomes reflect the specific payoffs that result from the course of action taken by the physician.

The decision tree shows the costs and benefits that flow from alternate actions taken by the physician. In this regard, $C_{E}$ is the cost of a medical exam, $C_{A}$ is the cost of an antibiotic, $C_{A R}$ is the societal cost of antibiotic resistance, and $C_{C}$ is the cost incurred if a medical complication arises. Note that although all of these costs do not directly affect the physician, it is important to include them in the analysis because the physician must consider these costs in order to treat the patient effectively. The preceding four costs are all time-invariant. Coming to benefits, $B$ (treatment) is the benefit when a bacterial infection is treated successfully and $B$ (no infection) is the benefit stemming from the physician's diagnosis that the patient has no bacterial infection. Note that even when the patient is diagnosed to have no bacterial infection, she may still have a viral infection. The probability $P_{S}$ is the likelihood that the patient is infected when she comes to the physician's office and this probability is assumed to be a function of the socioeconomic circumstances confronting the physician. Finally, $P_{A R}$ is the probability of developing antibiotic resistance. The payoffs to the physician from the five possible outcomes numbered 1 through 5 in Figure 1 are shown in detail in Table 1. I shall now analyze these payoffs in greater detail.

\subsection{Numerical Analysis}

In this section, I shall focus on the impact of numerical changes in the infection probability $P_{S}$ only. This is because I am interested in understanding how ${ }^{3}$ It is sometimes possible to treat a bacterial infection with a non-antibiotic treatment option. 


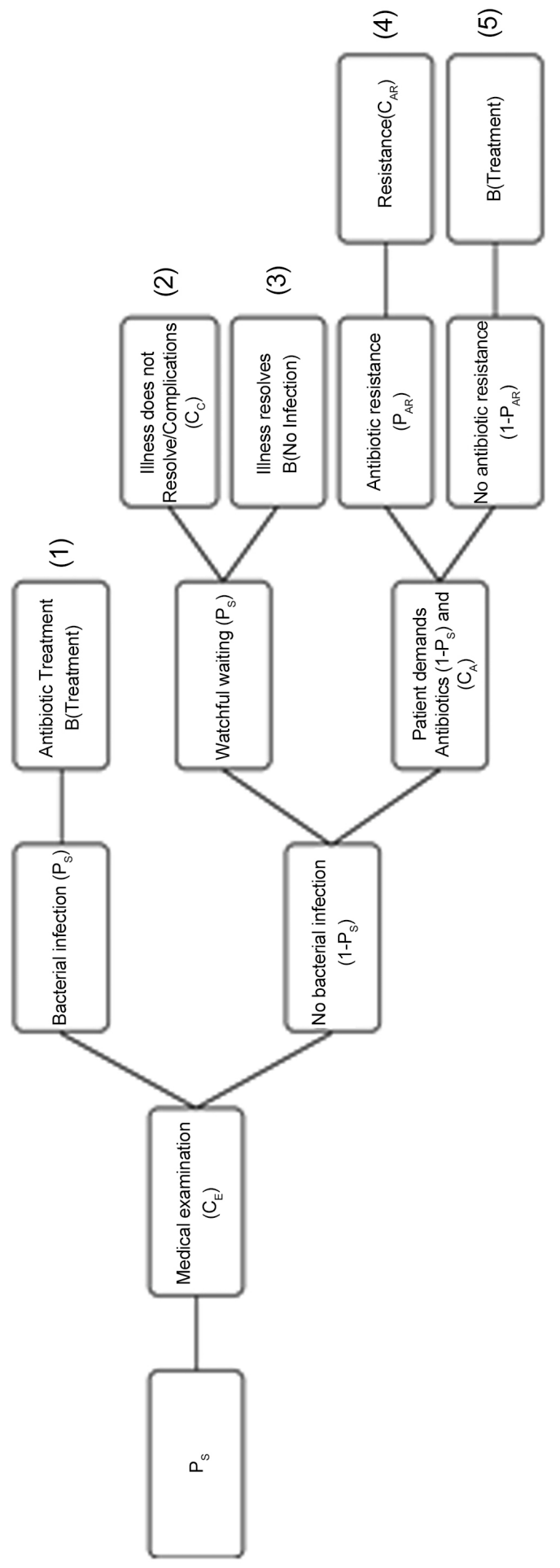

Figure 1. Physician's decision tree.

socioeconomic and demographic factors-that affect $P_{S}$-influence decision-making by the physician. Inspection of Table 1 shows that $P_{S}$ appears in all five payoffs numbered 1 through 5 . Therefore, by focusing specifically on the impact of changes in $P_{S}$, I am able to examine how numerical changes in this 
Table 1. Payoffs to the physician in alternate circumstances.

\begin{tabular}{cc}
\hline & Read the right most boxes in Figure 1 from top to bottom \\
\hline 1 & $C_{E}+P_{S}[B($ treatment $)]$ \\
2 & $C_{E}+\left(1-P_{S}\right) P_{S} C_{C}$ \\
4 & $C_{E}+\left(1-P_{S}\right) P_{S}[B($ noinfection $)]$ \\
5 & $C_{E}+\left[\left(1-P_{S}\right)^{2}\left(C_{A}\right)\right]\left[\left(P_{A R}\right)\left(C_{A R}\right)\right]$ \\
\hline
\end{tabular}

probability affect all possible outcomes for the physician and not just a few outcomes.

As noted above, the probability $P_{S}$ is a function of both economic $(E)$ and sociological $(S)$ variables. I can express this relationship mathematically as $P_{S}=f(E, S)$. In addition, the reader should think of $P_{S}$ as a probability that denotes the socioeconomic status confronting a physician. I now illustrate the working of my model by picking three numerical values for the key exogenous variable of interest, i.e., $P_{S}$. I pick $P_{S}=0.2,0.5,0.8$. In addition, I have chosen fixed values for:

1) The other probability $P_{A R}=0.2$,

2) The cost terms $\left(C_{A}=10, C_{E}=8.5, C_{C}=50, C_{A R}=100\right)$, and

3) The benefit terms $(B($ no infection $)=-25, B($ treatment $)=-25)$. These values are shown in Table 2. Substituting these numerical values into the various payoffs shown in Table 1, I obtain the five numerical payoffs to the physician shown in Table 3.

I picked a low value (0.2), a middle value (0.5), and a high value (0.8) for the probability $P_{S}$ in Table 2 to illustrate the impact of changes in $P_{S}$ across the entire range of $P_{S}$. As for the numerical choices for the other variables in Table 2, the reasoning supporting my choices is as follows. The cost $C_{A R}=100$ because $C_{A R}$ represents the social cost of antibiotic resistance. This value would be lower if one were concerned only about the private cost of antibiotic resistance.

To see why the two benefit terms in Table 2 are negative, note the following three points. First, the payoff expressions are written in terms of costs and benefits and they both enter the individual expressions with positive signs. Second, it is clear that a cost is "a bad" and a benefit is "a good." Third, when we think of the individual payoffs in terms of expected net benefits, the benefit terms would be positive and the cost terms would be negative. However, if we think of the individual payoffs in terms of expected net costs-which is what I am doing-then the cost terms would be positive and the benefit terms would be negative.

The cost of complications $C_{C}$ is greater than the cost of the antibiotic $C_{A}$ because it is generally more expensive to seek medical treatment for a more serious illness than for a relatively minor one. Put differently, it is more expensive to provide urgent care in an emergency room than to provide care in a primary care physician's office. 
Table 2. Numerical inputs for the physician's payoffs.

\begin{tabular}{cccccccc}
\hline$P_{S}$ & $P_{A R}$ & $C_{A}$ & $C_{E}$ & $C_{C}$ & $C_{A R}$ & $B$ (noinfection) & $B$ (treatment) \\
\hline 0.2 & 0.2 & 10 & 8.5 & 50 & 100 & -25 & -25 \\
0.5 & 0.2 & 10 & 8.5 & 50 & 100 & -25 & -25 \\
0.8 & 0.2 & 10 & 8.5 & 50 & 100 & -25 & -25 \\
\hline
\end{tabular}

Table 3. Numerical outputs for the physician's payoffs ( ${ }^{*}$ indicates lowest expected net cost).

\begin{tabular}{cccccc}
\hline$P_{S}$ & Payoff 1 & Payoff 2 & Payoff 3 & Payoff 4 & Payoff 5 \\
\hline 0.2 & 3.5 & 16.5 & 4.5 & 136.5 & $-119.5^{*}$ \\
0.5 & -4 & 21 & 2.25 & 58.5 & $-41.5^{*}$ \\
0.8 & $-11.5^{*}$ & 16.5 & 4.5 & 16.5 & 0.5 \\
\hline
\end{tabular}

From the outputs shown in Table 3, the lowest expected net cost option for $P_{S}=0.2$ and $P_{S}=0.5$ is payoff 5 . This payoff arises when the physician believes that the patient does not have a bacterial ear infection but the parent demands an antibiotic, and it turns out that the doctor misdiagnosed the cause of the ear infection. However, when $P_{S}=0.8$, the lowest expected net cost option switches to payoff 1 . This payoff arises when the doctor diagnoses the source of the ear infection as a bacterial infection and then prescribes the appropriate antibiotic. Note that there exists a value of $P_{S}$ in the open interval $(0.5,0.8)$ that results in a switch of the lowest expected net cost to the physician. I now provide a more detailed qualitative discussion of the physician's medical decision-making with particular emphasis on the infection probability $P_{S}$.

\section{Qualitative Analysis}

\subsection{An Interpretation of the Infection Probability}

I now focus on a "real-world" interpretation of $P_{S}$ from the physician's perspective. To begin the discussion, consider first the physician's physical location, i.e., whether his clinic is situated in a rich or a poor area. I want to concentrate on the implications of having a clinic located in a poor as opposed to a rich area. Specifically, I want to relate the dichotomous geographic location to the previously mentioned notion of socioeconomic status (SES). To this end, I assume that physicians with clinics in poor (rich) areas treat patients that are mainly of low (high) SES. This assumption is supported by the findings of prior studies in the literature. For instance, Kumar et al. [9] study the prescription of antibiotics for sore throats by physicians in the United Kingdom. They point out that general practitioners serving people living in low-income housing, in overcrowded conditions with poor nutrition, or substance abuse (including alcohol), thought that these factors compromised a person's immune function and hence increased their susceptibility to bacterial complications. In other words, the presence of adverse socioeconomic factors lowered a general practitioner's threshold 
for prescribing antibiotics for a sore throat.

This finding implies, in part, that some physicians may be unnecessarily diagnosing their patients with bacterial infections and thus prescribing antibiotics because they believe that the living conditions of these patients is likely to increase the likelihood of a bacterial infection. The point to note is that a physician's (potentially inaccurate) belief about patients with lower SES reduces his threshold for prescribing an antibiotic. Payoffs 4 and 5 in Table 1 and Table 3 represent the situation I have just mentioned. Specifically, the numerical analysis I presented in Section 2.2 shows that payoff 5 denotes the optimal course of action for a physician who believes that the infection probability $P_{S}=0.2$ or $P_{S}=0.5$.

Given the above discussion, the reader should note that physicians serving underprivileged patients in a clinic in a relatively poor area are more likely to make errors in their decision-making than their counterparts working in clinics located in wealthy areas and serving people with high SES. I suppose that these "more vulnerable to error" physicians-who frequently work with fewer resources-are more likely to believe that the likelihood of an infection is lower than it actually is. As such, I shall represent such physicians with the infection probabilities of $P_{S}=0.2$ and $P_{S}=0.5^{4}$. In contrast, I suppose that the physicians operating clinics in relatively wealthy areas are more accurately able to determine the likelihood of an infection when there is an infection to contend with. I shall represent these physicians-see Figure 2-with the infection probability $P_{S}=0.8$. For such physicians, the optimal course of action is the one leading to payoff 1 where the patient has a bacterial ear infection and is therefore treated with an antibiotic.

\subsection{The Patient-Physician Relationship}

The nature of the relationship between a patient and her physician has a great bearing on the question of antibiotic resistance. Existing studies by Barden et al. [19], Butler et al. [11], Cockburn and Pit [20], and Mangione-Smith et al. [10] show that physicians are greatly influenced by the implicit or the explicit expectations of their patients. In particular, there is a sinister outcome that results from physicians feeling pressured to prescribe antibiotics to their patients and that outcome is this: when a sick patient expects an antibiotic and a physician "satisfies" this expectation by prescribing an antibiotic even when it is unnecessary, this action reinforces the patient's belief that an antibiotic is necessary to cure her of her ailment. This means that the next time the patient has a similar ailment, her expectation that she needs an antibiotic will be strengthened by her physician's past actions, and hence this physician will feel even further pressured to prescribe an antibiotic. As noted by Butler et al. [11], this unfortunate state of ${ }^{4}$ In principle, it is possible that such physicians believe that the likelihood of an infection is greater than it actually is. However, if this were the case then we ought to be observing excessive medication of underprivileged patients who visit clinics in poorer areas but this something that we typically do not observe. Hence I omit any further discussion of this case. 


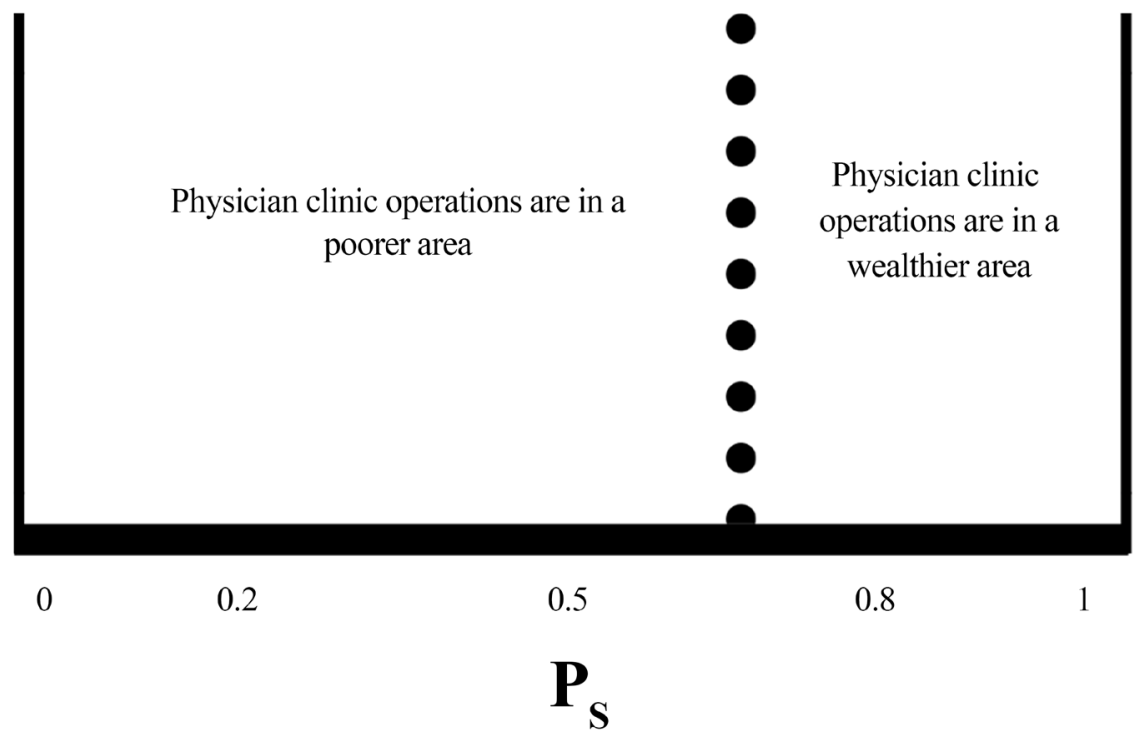

Figure 2. Relationship between a physician's clinic location and the infection probability $P_{S}$

affairs creates a vicious cycle that is difficult to get out of.

This vicious cycle is well demonstrated in the decision-making behavior of older versus younger physicians. Butler et al. [11] and Kumar et al. [9] both point out that as physicians age, their proclivity for prescribing an antibiotic tends to increase. It is possible to relate this finding in the literature to the physician's decision tree and in particular to the infection probability $P_{S}$ I have been discussing thus far. In this regard, consider a young physician who has no long established group of patients and is therefore less vulnerable to the existing expectations of this group. As such, relative to an older physician, he is more accurately able to determine when a particular patient's ailment calls for treatment with an antibiotic. This means that the infection probability $P_{S}$ is relatively high for such a physician. Now consider an old physician. Reversing the above logic, this physician is significantly more beholden to the expectations of his patient group and hence this older physician's ability to accurately determine when an antibiotic is needed can be thought of as being lower than the ability of a younger physician. This means that $P_{S}$ is relatively low for such a physician. Consistent with this discussion and as shown in Figure 3, one can associate $P_{S}=0.8$ with a young physician and $P_{S}=0.2$ and $P_{S}=0.8$ with an old physician. This completes my discussion of medical decision-making by a physician in the presence of resistance to antibiotics.

\section{Conclusions}

Antibiotic resistance is an enormous threat to humanity and it ought to be regarded as a public health crisis. Despite the fact that experts in the field have been discussing this matter for over two decades, the abuse of antibiotics is still an issue today. In fact, as Nyquist et al. [21] have noted, children receive a 


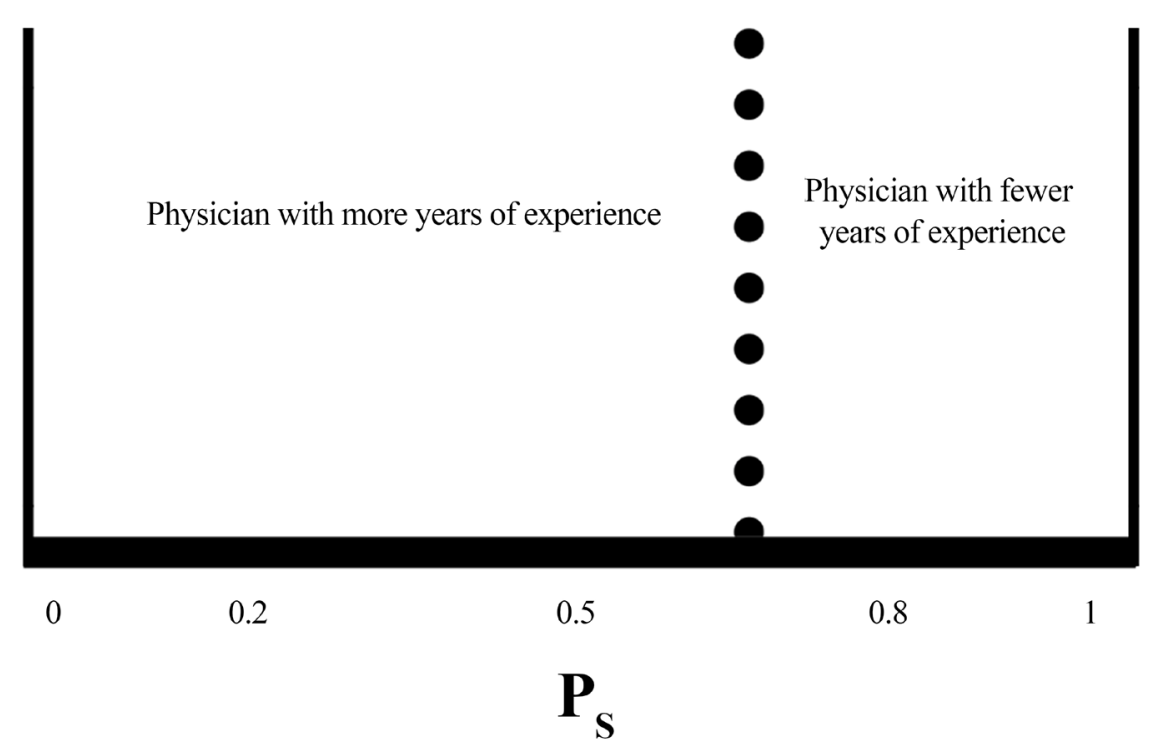

Figure 3. Relationship between a physician's years of experience and the infection probability $P_{S}$.

significant portion of the total prescribed antibiotics. Given this state of affairs, in this paper, I used the concept of a decision tree to shed light on how socioeconomic factors influenced medical decision-making by physicians when antibiotic resistance was an issue to contend with.

The analysis contained in this paper can be extended in a number of different directions. Here are four examples of such extensions. First, the decision tree based analysis in this paper is static. As such, it would be useful to analyze the patient-physician interaction in a dynamic setting where they interact repeatedly over time. Second, the numerical analysis conducted in this paper is based on the use of fictitious data. Therefore, it would be instructive to collect data and then determine the extent to which my decision tree based approach can be operationalized in a given real-world setting. Third, it may also be useful to use a game-theoretic approach as in Greenberg et al. [22] to study the patient-physician interaction. Finally, a hybrid model that allows for the use of both antibiotic and non-antibiotic treatment options in the same theoretical framework may yield additional insights into patient-physician interactions. Studies that incorporate these aspects of the problem into the analysis will increase our understanding of the connections between patient expectations, medical decision-making by physicians, and the evolution of resistance to antibiotics.

\section{Acknowledgements}

I thank three anonymous reviewers and Robert Karpman for their helpful comments on a previous version of this paper. The usual disclaimer applies.

\section{Conflicts of Interest}

The author declares that she has no conflict of interest regarding the publication 
of this paper.

\section{References}

[1] Neu, H.C. (1992) The Crisis in Antibiotic Resistance, Science, 257, 1064-1073.

[2] Gardete, S. and Tomasz, A. (2014) Mechanisms of Vancomycin Resistance in Staphylococcus aureus. Journal of Clinical Investigation, 124, 2836-2840. https://doi.org/10.1172/JCI68834

[3] Elbasha, E.H. (2003) Deadweight Loss of Bacterial Resistance Due to Overtreatment. Health Economics, 12, 125-138. https://doi.org/10.1002/hec.702

[4] Fox, M. (2016) Drug-Resistant Superbug Are a "Fundamental Threat", WHO Says. NBC News.

https://www.nbcnews.com/health/health-news/who-labels-drug-resistant-superbugs -fundamental-threat-humans-n651981

[5] Centers for Disease Control and Prevention (CDC) (2017) Antibiotic Prescribing and Use in Doctor's Offices.

https://www.cdc.gov/antibiotic-use/community/for-patients/common-illnesses/earinfection.html

[6] Amábile-Ceuvas, C.F., Cárdenas-Garcia, M. and Ludgar, M. (1995) Antibiotic Resistance. American Scientist, 83, 320-329.

[7] McKenna, J. (1998) Natural Alternatives to Antibiotics. Avery Publishing Group, Garden City Park, NY.

[8] Centers for Disease Control and Prevention (CDC) (2018) Antibiotic/Antimicrobial Resistance. https://www.cdc.gov/drugresistance/index.html

[9] Kumar, S., Little, P. and Britten, N. (2003) Why Do General Practitioners Prescribe Antibiotics for Sore Throat? Grounded Theory Interview Study. British Medical Journal, 326, 138. https://doi.org/10.1136/bmj.326.7381.138

[10] Mangione-Smith, R., McGlynn, E.A., Elliott, M.N., Krogstad, P. and Brook, R.H. (1999) The Relationship between Perceived Parental Expectations and Pediatrician Antimicrobial Prescribing Behavior. Pediatrics, 103, 711-718.

https://doi.org/10.1542/peds.103.4.711

[11] Butler, C.C., Rollnick, S., Pill, R., Maggs-Rapport, F. and Stott, N. (1998) Understanding the Culture of Prescribing: Qualitative Study of General Practitioners' and Patients' Perceptions of Antibiotics for Sore Throats. British Medical Journal, 317, 637-642. https://doi.org/10.1136/bmj.317.7159.637

[12] Fox, M. (2018) "Nightmare Bacteria" Are Trying to Spread in the U.S., CDC Says. NBC News.

https://www.nbcnews.com/health/health-news/nightmare-bacteria-are-trying-sprea d-u-s-cdc-says-n862436

[13] Bornstein, B.H. and Emler, A.C. (2001) Rationality in Decision Making: A Review of the Literature on Doctors' Decision Making Biases. Journal of Evaluation in Clinical Practice, 7, 97-107. https://doi.org/10.1046/j.1365-2753.2001.00284.x

[14] Macfarlane, J., Holmes, W., Macfarlane, R. and Britten, N. (1997) Influence of Patients' Expectations on Antibiotic Management of Acute Lower Respiratory Tract Illness in General Practice: Questionnaire Study. British Medical Journal, 315, 1211-1214. https://doi.org/10.1136/bmj.315.7117.1211

[15] Rollnick, S., Seale, C., Rees, M., Butler, C., Kinnersley, P. and Anderson, L. (2001) Inside the Routine General Practice Consultation: And Observation Study of Consultations for Sore Throats. Family Practice, 18, 506-510. 
https://doi.org/10.1093/fampra/18.5.506

[16] Manzo, G. (2007) The Progress and "Urgency" of Modeling in Sociology. The Concept of "Generative Modeling" and Its Implementation, L’Année sociologique, 57, 13-61.

[17] Batabyal, S.S. and Batabyal, A.A. (2018) Medical Decision-Making by Patients and Providers under Uncertainty and in the Presence of Antibiotic Resistance. Forthcoming. International Review of Economics and Finance.

[18] Ross, S.M. (2003) Introduction to Probability Models. $8^{\text {th }}$ Edition, Academic Press, San Diego, CA.

[19] Barden, L.S., Dowell, S.F., Schwartz, B. and Lackey, S. (1998) Current Attitudes Regarding Use of Antimicrobial Agents: Results from Physicians' and Parents' Focus Group Discussions. Clinical Pediatrics, 37, 665-671. https://doi.org/10.1177/000992289803701104

[20] Cockburn, J. and Pit, S.W. (1997) Prescribing Behavior in Clinical Practice: Patients' Expectations and Doctors' Perceptions of Patients' Expectations: A Questionnaire Study. British Medical Journal Clinical Research, 315, 520-523.

https://doi.org/10.1136/bmj.315.7107.520

[21] Nyquist, A., Gonzalez, R., Steiner, J.F. and Sande, M.A. (1998) Antibiotic Prescribing for Children with Colds, Upper Respiratory Tract Infections, and Bronchitis. Journal of the American Medical Association, 279, 875-877. https://doi.org/10.1001/jama.279.11.875

[22] Greenberg, J., Luo, X., Oladi, R. and Shitovitz, B. (2002) (Sophisticated) Stable Sets in Exchange Economies. Games and Economic Behavior, 39, 54-70.

https://doi.org/10.1006/game.2001.0890 